\title{
A PARAMETRIZATION OF SOLUTIONS OF THE DISCRETE-TIME ALGEBRAIC RICCATI EQUATION BASED ON PAIRS OF OPPOSITE UNMIXED SOLUTIONS*
}

\author{
HARALD K. WIMMER ${ }^{\dagger}$
}

\begin{abstract}
The paper describes the set of solutions of the discrete-time algebraic Riccati equation. It is shown that each solution is a combination of a pair of opposite unmixed solutions. There is a one-to-one correspondence between solutions and invariant subspaces of the closed loop matrix of an unmixed solution. The results of the paper provide an extended counterpart of the parametrization theory of continuous-time algebraic Riccati equations by Willems, Coppel, and Shayman.
\end{abstract}

Key words. discrete-time algebraic Riccati equation, unmixed solutions, opposite solutions, parametrization by invariant subspaces, shorted operators, Schur complements

AMS subject classifications. 15A24, 93C55, 47A64

DOI. $10.1137 /$ S0363012904441362

1. Introduction. To a large extent the groundwork for the investigation of algebraic Riccati equations by geometric methods was laid by J. C. Willems. In his study of least squares stationary optimal control [24], he gave a complete description of the set of symmetric solutions of the real continuous-time algebraic Riccati equation (CARE)

$$
-F^{T} X-X F+X G G^{T} X-Q=0 .
$$

Assuming that $(F, G)$ is controllable and that the associated Hamiltonian matrix

$$
H=\left[\begin{array}{cc}
F & -G G^{T} \\
-Q & -F^{T}
\end{array}\right]
$$

has no eigenvalues on the imaginary axis, Willems showed that (1.1) has a greatest solution $X^{+}$and a least solution $X^{-}$. The eigenvalues of the corresponding closed loop matrix $F_{X^{+}}=F-G G^{T} X^{+}$lie in the left half-plane and those of $F_{X^{-}}$are in the right half-plane. Moreover, according to [24] there is a one-to-one correspondence of solutions of (1.1) and invariant subspaces of $F_{X^{+}}$such that every solution of (1.1) can be expressed as a combination of $X^{+}$and $X^{-}$. That result was refined by Coppel [5] and extended further by Shayman [23].

In this paper we consider the complex discrete-time algebraic Riccati equation (DARE)

$$
X-F^{*} X F+\left(G^{*} X F+S\right)^{*}\left(R+G^{*} X G\right)^{-1}\left(G^{*} X F+S\right)-Q=0 .
$$

We shall obtain a classification of solutions of (1.2) that corresponds to the WillemsCoppel-Shayman parametrization for (1.1).

Let us first recapitulate the parametrization result for the CARE (1.1). It will be assumed that elementary divisors of $H$ have even degree when they belong to pure

\footnotetext{
* Received by the editors February 24, 2004; accepted for publication (in revised form) June 3, 2005; published electronically January 6, 2006.

http://www.siam.org/journals/sicon/44-6/44136.html

${ }^{\dagger}$ Mathematisches Institut, Universität Würzburg, Am Hubland, D-97074 Würzburg, Germany (wimmer@mathematik.uni-wuerzburg.de).
} 
imaginary eigenvalues. A solution $X$ of (1.1) is called unmixed [23] if the spectrum of $F_{X}=F-G G^{T} X$ satisfies

$$
\sigma\left(F_{X}\right) \cap \sigma\left(-F_{X}^{T}\right) \subseteq i \mathbb{R} .
$$

It is known (see, e.g., [12]) that there exists an unmixed solution of (1.1) if and only if the pair $(F, G) \in \mathbb{R}^{n \times n} \times \mathbb{R}^{n \times p}$ is sign-controllable, i.e., if $\lambda \in \sigma(F)$ and $\operatorname{rank}[\lambda I-F, G]<n$ imply $\operatorname{rank}[-\bar{\lambda} I-F, G]=n$. Let $X_{1}$ be an unmixed solution of (1.1). Then there exists a unique (unmixed) solution $X_{2}$ such that $F_{X_{1}}$ and $F_{X_{2}}$ have, at most, pure imaginary eigenvalues in common if and only if $(F, G)$ is controllable. In that case $\left(X_{1}, X_{2}\right)$ is called a pair of opposite unmixed solutions [23]. The extremal solutions $\left(X^{-}, X^{+}\right)$are an example of such a pair.

Let $\operatorname{Inv}(F)$ denote the set of $F$-invariant subspaces of $\mathbb{R}^{n}$. If $i \alpha$ is an eigenvalue of $F$ on the imaginary axis, we set $E_{ \pm i \alpha}=\operatorname{Ker}\left(F^{2}+\alpha^{2} I\right)^{n}$ and we define

$$
E_{i \mathbb{R}}(F)=\underset{\alpha \in \mathbb{R}}{\oplus} E_{ \pm i \alpha}
$$

The following theorem can be regarded as an updated version of the Willems-CoppelShayman theory. It combines results of [24], [5], and [23] with an approach by Scherer $[22]$ and observations on shorted operators in section 3.

THEOREM 1.1. Let $\Gamma$ be the set of real symmetric solutions of (1.1). Assume that $(F, G)$ is controllable. Let $\left(X_{1}, X_{2}\right)$ be a pair of opposite unmixed solutions of

$$
-F^{T} X-X F+X G G^{T} X-Q=0,
$$

and let $F_{X_{2}}=F-G G^{T} X_{2}$ be the closed loop matrix corresponding to $X_{2}$. Set $\Delta=X_{1}-X_{2}$. Define

$$
\mathcal{N}=\left\{N \in \operatorname{Inv}\left(F_{X_{2}}\right) \mid E_{i \mathbb{R}}\left(F_{X_{2}}\right) \subseteq N\right\} .
$$

If $N \in \mathcal{N}$, then

$$
(\Delta N)^{\perp} \oplus(N \cap \operatorname{Im} \Delta)=\mathbb{R}^{n} .
$$

Let $P_{N}$ be the projection on $(\Delta N)^{\perp}$ along $(N \cap \operatorname{Im} \Delta)$. Define

$$
\kappa(N)=X_{1} P_{N}+X_{2}\left(I-P_{N}\right) .
$$

Then $\kappa: \mathcal{N} \rightarrow \Gamma$ is a bijection. If $X \in \Gamma$, then

$$
\kappa^{-1}(X)=\operatorname{Ker}\left(X-X_{2}\right) .
$$
form

It is not difficult to explain (see also [18]) why direct sum decompositions of the

$$
(\Delta N)^{\perp} \oplus N=\mathbb{R}^{n}
$$

and associated projections $P_{N}$ should play a role in the theory of the CARE. Consider (1.1) with $Q=0$, i.e.,

$$
\mathcal{C}(X)=-F^{T} X-X F+X G G^{T} X=0 .
$$


If $(F, G)$ is controllable and $\sigma(F) \cap \sigma\left(-F^{T}\right)=\emptyset$, then (1.5) has a unique nonsingular solution $\Delta$ (see, e.g., $\left[22\right.$, p. 102]). Let $N=\operatorname{Im}(I, O)^{T}$ be invariant under $F$ and let $\Delta$ be partitioned according to $F$ such that

$$
F=\left[\begin{array}{cc}
F_{1} & F_{12} \\
0 & F_{2}
\end{array}\right] \text { and } \Delta=\left[\begin{array}{cc}
\Delta_{1} & \Delta_{12} \\
\Delta_{12}^{T} & \Delta_{2}
\end{array}\right]
$$

Then $\Delta_{1}$ is nonsingular [22]. Let $\widetilde{\Delta}_{2}=\Delta_{2}-\Delta_{12}^{T} \Delta_{1}^{-1} \Delta_{12}$ be the Schur complement of $\Delta$ with respect to $N$. Set

$$
S=\left[\begin{array}{cc}
I & -\Delta_{1}^{-1} \Delta_{12} \\
0 & I
\end{array}\right]
$$

Then $S^{T} \Delta S=\operatorname{diag}\left(\Delta_{1}, \widetilde{\Delta}_{2}\right)$ and

$$
S^{-1} F S=\left[\begin{array}{cc}
F_{1} & * \\
0 & F_{2}
\end{array}\right] .
$$

It is obvious that

$$
\widetilde{\Delta}=\left[\begin{array}{cc}
0 & 0 \\
0 & \widetilde{\Delta}_{2}
\end{array}\right]
$$

is a solution of (1.5). In the terminology of [2], [16], or [6], the matrix $\widetilde{\Delta}$ is a shorted matrix of $\Delta$. In section 3 we shall give a basis-free description of $\widetilde{\Delta}$ and we shall see that $\widetilde{\Delta}=\Delta P_{N}$, where $P_{N}$ is the projection corresponding to (1.4).

Notation: Let $\mathbb{D}$ be the open unit disk and $\partial \mathbb{D}=\{z \in \mathbb{C} ;|z|=1\}$ be the unit circle. Let $\Lambda$ be a set of complex numbers and define

$$
\Lambda^{\nabla}=\left\{\bar{\lambda}^{-1} \mid \lambda \in \Lambda, \lambda \neq 0\right\}
$$

To a matrix of complex rational functions of the form $W(s)=D+C(s I-A)^{-1} B$, we associate $W^{\nabla}(s)=D^{*}+B^{*}\left(s^{-1} I-A^{*}\right) C^{*}$. For $\lambda \in \mathbb{C}$ put

$$
E_{\lambda}(F)=\operatorname{Ker}(\lambda I-F)^{n} .
$$

Then $E_{\lambda}(F)$ is a generalized eigenspace of $F$ if $\lambda \in \sigma(F)$. In particular, $E_{0}(F)=$ Ker $F^{n}$. We set

$$
E_{\partial \mathbb{D}}=\oplus\left\{E_{\eta}(F), \eta \in \partial \mathbb{D}\right\} .
$$

A subspace $V$ of $\mathbb{C}^{n}$ is a spectral subspace of $F$ if $V=\oplus\left\{E_{\lambda}(F) ; \lambda \in T\right\}$ for some $T \subseteq \sigma(F)$. If $K$ is a hermitian matrix, we write $K>0(K \geq 0)$ when $K$ is positive (semi)definite. If $F$ is nonsingular, we set $F^{-*}=\left(F^{*}\right)^{-1}$.

The main result of this paper is Theorem 5.3. It describes a parametrization of the set of hermitian solutions of the DARE (1.2). The proof of that theorem will proceed in several stages. In section 2 we review basic facts on the DARE. We recall that the difference of two solutions of (1.2) satisfies an associated DARE of the form

$$
X-F^{*} X F+F^{*} X G\left(R+G^{*} X G\right)^{-1} G^{*} X F=0 .
$$

Such equations, where $X=0$ is a solution, will be considered in section 4 . We have indicated before that (1.4) is related to Schur complements and shorted operators. That subject will be touched upon in section 3 .

The geometric theory of Willems [24] and Coppel [5] was carried over to the DARE (1.2) first by G. Ruckebusch [20], [21, p. 129] and then by Ran and Trentelman [19]. Those papers give a geometric characterization of the set of hermitian solutions in terms of the pair of extremal solutions. 
2. Basic facts of the DARE: Definitions. There is a wide class of problems in systems and control theory that require solutions of the DARE

$$
\mathcal{D}(X)=X-F^{*} X F+\left(G^{*} X F+S\right)^{*}\left(R+G^{*} X G\right)^{-1}\left(G^{*} X F+S\right)-Q=0 .
$$

An important example is the discrete-time linear quadratic problem of optimal control. Let the system

$$
x(t+1)=F x(t)+G u(t), x(0)=x_{0}
$$

be stabilizable, and let

$$
J\left(x_{0}, u\right)=\sum_{t=0}^{\infty}\left[x^{*}(t) u^{*}(t)\right]\left[\begin{array}{cc}
Q & S^{*} \\
S & R
\end{array}\right]\left[\begin{array}{l}
x(t) \\
u(t)
\end{array}\right]
$$

be a positive semidefinite performance index. Then (see, e.g., [9]) there exists an optimal control $u(t)$ which minimizes $J\left(x_{0}, u\right)$. The optimal cost is given by $J_{\text {opt }}=$ $x_{0}^{*} X_{\mathrm{opt}} x_{0}$, where $X_{\mathrm{opt}}$ is the smallest positive semidefinite solution of (2.1), and the optimal control is

$$
u_{\mathrm{opt}}(t)=-\left(R+G^{*} X_{\mathrm{opt}} G\right)^{-1}\left(S+G^{*} X_{\mathrm{opt}} F\right) x(t) .
$$

Thus (2.2) gives rise to the closed loop system

$$
x(t+1)=\left[F-G\left(R+G^{*} X_{\mathrm{opt}} G\right)^{-1}\left(S+G^{*} X_{\mathrm{opt}} F\right)\right] x(t) .
$$

The matrices in (2.1) are assumed to be complex, $F \in \mathbb{C}^{n \times n}, G \in \mathbb{C}^{n \times m}, S \in$ $\mathbb{C}^{m \times n}, R=R^{*} \in \mathbb{C}^{m \times m}$, and $Q=Q^{*} \in \mathbb{C}^{n \times n}$. We are concerned with hermitian solutions $X$ of (2.1). In this section we assemble basic facts and concepts related to (2.1). With regard to (2.3) we define

$$
F_{X}=F-G\left(R+G^{*} X G\right)^{-1}\left(G^{*} X F+S\right)
$$

as the closed loop matrix corresponding to a solution $X$. We say that a solution $X$ of the DARE (2.1) is unmixed if $F_{X}$ has the property

$$
\sigma\left(F_{X}\right) \cap \sigma\left(F_{X}\right)^{\nabla} \subseteq \partial \mathbb{D}
$$

i.e., if $\lambda \in \sigma\left(F_{X}\right)$ and $\lambda \neq 0$ and $|\lambda| \neq 1$, then $\bar{\lambda}^{-1} \notin \sigma\left(F_{X}\right)$. We say that $\left(X_{1}, X_{2}\right)$ is a pair of opposite unmixed solutions if the corresponding closed loop matrices satisfy

$$
\sigma\left(F_{X_{1}}\right) \cap \sigma\left(F_{X_{2}}\right) \subseteq \partial \mathbb{D} \cup\{0\} .
$$

Let

$$
M-s L=\left[\begin{array}{ccc}
F & 0 & G \\
Q & I & S^{*} \\
S & 0 & R
\end{array}\right]-s\left[\begin{array}{ccc}
I & 0 & 0 \\
0 & F^{*} & 0 \\
0 & G^{*} & 0
\end{array}\right]
$$

be the extended symplectic pencil associated with (2.1), and let

$$
\Psi(s)=\left[G^{*}\left(s^{-1} I-F^{*}\right)^{-1} I\right]\left[\begin{array}{cc}
Q & S^{*} \\
S & R
\end{array}\right]\left[\begin{array}{c}
(s I-F)^{-1} G \\
I
\end{array}\right]
$$


be the associated Popov matrix. The following identities and facts can be found in [11], [13], and [17]. If $X$ is a solution of (2.1), then

$$
\operatorname{det}(M-s L)=\operatorname{det}\left(R+G^{*} X G\right) \operatorname{det}\left(s I-F_{X}\right) \operatorname{det}\left(I-s F_{X}^{*}\right) .
$$

It follows from (2.8) that there exists a solution of (2.1) only if $M-s L$ is nonsingular, i.e., if $\operatorname{det}(M-s L)$ is not the zero polynomial. In that case we call

$$
\sigma(M-s L)=\{\lambda \in \mathbb{C} \mid \operatorname{det}(M-\lambda L)=0\}
$$

the set of characteristic roots of the pencil (2.6). It is obvious from (2.8) that $\sigma\left(F_{X}\right) \subseteq$ $\sigma(M-s L)$. Hence, if 0 is a characteristic root of $M-s L$, then we have $0 \in \sigma\left(F_{X}\right)$ for all solutions $X$, which accounts for the singleton $\{0\}$ in (2.5). Let $X_{1}$ and $X_{2}$ be solutions of (2.1) such that (2.5) holds. Then it follows from (2.8) that both $X_{1}$ and $X_{2}$ are unmixed. Each solution $X$ of (2.1) gives rise to a factorization of the Popov matrix $\Psi(s)$, namely

$$
\Psi(s)=\Phi_{X}^{\nabla}(s)\left(R+G^{*} X G\right) \Phi_{X}(s)
$$

with

$$
\Phi_{X}(s)=I+\left(R+G^{*} X G\right)^{-1}\left(G^{*} X F+S\right)(s I-F)^{-1} G .
$$

If $\Psi(s)$ satisfies

$$
\Psi(\eta)>0 \text { for some } \eta \in \partial \mathbb{D}
$$

then (2.9) implies

$$
R+G^{*} X G>0
$$

for all solutions $X$.

Arguments in section 4 show that the conditions $(\Psi)$ and (2.10) are essential for the derivation of Theorem 5.3. Hence it is not within the scope of this paper to deal with DAREs of the form (1.2) where $\left(R+G^{*} X G\right)^{-1}$ is replaced by a generalized inverse $\left(R+G^{*} X G\right)^{\#}$.

Existence of unmixed solutions was studied in [4], [25], [3]. We note the following result of [3]. Suppose that $(\Psi)$ holds, $\Psi(\eta) \geq 0$ for almost all $\eta \in \partial \mathbb{D}$, and $\operatorname{rank}(F-$ $\lambda I)=n$ for all $\lambda \neq 0$. Then each unmixed set $\Lambda$ gives rise to a unique solution $X$ such that $\sigma\left(F_{X}\right) \subseteq \Lambda$. In particular there exists a unique pair $\left(X_{-}, X_{+}\right)$such that

$$
\sigma\left(F_{X_{-}}\right) \subseteq\{0\} \cup\{\lambda \in \mathbb{C} ;|\lambda| \geq 1\} \text { and } \sigma\left(F_{X_{+}}\right) \subseteq \overline{\mathbb{D}}
$$

It is known that Cayley transformations allow a passage from continuous-time to discrete-time algebraic Riccati equations [15], [1]. The use of such transformations requires invertibility assumptions (see, e.g., $[1$, p. 81]) which are not met by the general hypotheses in the present paper. We note that in the case of $0 \in \sigma(M-s L)$ a computational procedure is available [7] to obtain an equivalent DARE of smaller order such that the associated closed loop matrices are nonsingular. 
3. Shorted operators and oblique projections. Let $N$ be a subspace of $\mathbb{C}^{n}$ and $\Delta$ be a hermitian $n \times n$ matrix.

LEMma 3.1. (i) If

$$
N=\operatorname{Im}\left[\begin{array}{c}
I_{t} \\
0
\end{array}\right], \quad \text { and if } \quad \Delta=\left[\begin{array}{cc}
\Delta_{1} & \Delta_{21}^{*} \\
\Delta_{21} & \Delta_{2}
\end{array}\right]
$$

is partitioned conformably, then we have

$$
\mathbb{C}^{n}=(\Delta N)^{\perp} \oplus N
$$

if and only if $\Delta_{1}$ is nonsingular.

(ii) Assume (3.2) and let $P_{N}$ be the projection on $(\Delta N)^{\perp}$ along $N$. Then the matrix $\Delta P_{N}$ is hermitian. If $N$ and $\Delta$ are given as in (3.1), then

$$
\Delta P_{N}=\operatorname{diag}\left(0, \tilde{\Delta}_{2}\right), \quad \tilde{\Delta}_{2}=\Delta_{2}-\Delta_{21}^{*} \Delta_{1}^{-1} \Delta_{21} .
$$

Proof. (i) Suppose det $\Delta_{1}=0$. If $u_{1} \in \operatorname{Ker} \Delta_{1}, u_{1} \neq 0$, then

$$
u=\left[\begin{array}{c}
u_{1} \\
0
\end{array}\right] \in N
$$

and

$$
u^{*} \Delta N=\left[\begin{array}{ll}
u_{1}^{*} & 0
\end{array}\right]\left[\begin{array}{c}
\Delta_{1} \\
\Delta_{21}
\end{array}\right]=0 .
$$

Hence $(\Delta N)^{\perp} \cap N \neq 0$. Conversely, if $\Delta_{1}$ is nonsingular, then

$$
\Delta N=\operatorname{Im}\left[\begin{array}{c}
\Delta_{1} \\
\Delta_{21}
\end{array}\right]=\operatorname{Im}\left[\begin{array}{c}
I_{t} \\
\Delta_{21} \Delta_{1}^{-1}
\end{array}\right]
$$

and

$$
(\Delta N)^{\perp}=\operatorname{Im}\left[\begin{array}{c}
-\Delta_{1}^{-1} \Delta_{21}^{*} \\
I_{n-t}
\end{array}\right]
$$

imply (3.2). (ii) From $\operatorname{Im} P_{N}=(\Delta N)^{\perp}$ and $\Delta \operatorname{Im}\left(I-P_{N}\right)=\Delta N$ follows $\left(I-P_{N}\right)^{*} \Delta P_{N}=0$. Hence, $\Delta P_{N}=P_{N}^{*} \Delta P_{N}$ is hermitian. Because of (3.4) the projection matrix $P_{N}$ is given by

$$
P_{N}=\left[\begin{array}{cc}
0 & -\Delta_{1}^{-1} \Delta_{21}^{*} \\
0 & I_{n-t}
\end{array}\right]
$$

which yields $\Delta P_{N}$ as a shorted operator in block diagonal form (3.3).

The following observation is a special case of a formula for the inverse of a block matrix $[10$, p. 18]. It will be needed in the proof of Theorem 4.5 , which deals with a nonsingular solution $\Delta$ of a special DARE and its inverse.

Lemma 3.2. Assume that $\Delta$ is nonsingular, set $W=\Delta^{-1}$, and let

$$
\Delta=\left[\begin{array}{cc}
\Delta_{1} & \Delta_{21}^{*} \\
\Delta_{21} & \Delta_{2}
\end{array}\right] \text { and } W=\left[\begin{array}{cc}
* & * \\
* & W_{2}
\end{array}\right]
$$

be partitioned accordingly. (i) Then $\Delta_{1}$ is nonsingular if and only if $W_{2}$ is nonsingular. (ii) If $\Delta_{1}$ is nonsingular, then $\widetilde{\Delta}_{2}=\Delta_{2}-\Delta_{21}^{*} \Delta_{1}^{-1} \Delta_{21}$ is nonsingular and $\widetilde{\Delta}_{2}^{-1}=W_{2}$. 
Proof. We include a proof which is an application of the preceding lemma. Set $N=\operatorname{Im}\left[\begin{array}{ll}I_{t} & O\end{array}\right]^{T}$ and $M=N^{\perp}$. (i) Assume that $\Delta_{1}$ is nonsingular, or equivalently (3.2). Because of $(\Delta N)^{\perp}=\Delta^{-1} N^{\perp}$ we can write (3.2) as $W M \oplus M^{\perp}=\mathbb{C}^{n}$, which in turn is equivalent to $(W M)^{\perp} \oplus M=\mathbb{C}^{n}$. Because of $M=\operatorname{Im}\left[\begin{array}{ll}O I_{n-t}\end{array}\right]^{T}$ such a decomposition exists if and only if $W_{2}$ is nonsingular. (ii) From $\Delta P_{N}=\operatorname{diag}\left(0, \widetilde{\Delta}_{2}\right)$ follows

$$
\Delta P_{N} \Delta^{-1}=\left[\begin{array}{cc}
0 & 0 \\
* & \widetilde{\Delta}_{2} W_{2}
\end{array}\right]
$$

Obviously $\Delta P_{N} \Delta^{-1}$ is the projection on $N^{\perp}$ along $\Delta N$. Thus we obtain $\widetilde{\Delta}_{2} W_{2}=$ I. $\quad \square$

Let $K=\operatorname{Ker} \Delta$ and $V=\operatorname{Im} \Delta$. We assume $0<n_{c}=\operatorname{dim} V<n$ such that the decomposition $\mathbb{C}^{n}=K \oplus V$ is nontrivial. Let $U \subseteq V$. Then $U^{\perp_{c}}$ shall denote the orthogonal complement of $U$ with respect to $V$. Set $\Delta_{c}=\Delta_{\mid V}$. Then $\Delta_{c}: V \rightarrow V$ is hermitian and nonsingular.

Lemma 3.3. Let $N$ be a subspace of $\mathbb{C}^{n}$ with $K \subseteq N$. Set $N_{c}=N \cap V$. Then

$$
\left(\Delta_{c} N_{c}\right)^{\perp_{c}} \oplus N_{c}=V
$$

if and only if

$$
(\Delta N)^{\perp} \oplus(N \cap \operatorname{Im} \Delta)=\mathbb{C}^{n} .
$$

Proof. Note that $(\Delta N)^{\perp}=K \oplus\left(\Delta_{c} N_{c}\right)^{\perp_{c}}$ and $\quad N \cap \operatorname{Im} \Delta=N_{c}$.

4. A DARE with solution $\boldsymbol{X}=\mathbf{0}$. In this section we deal with the equation

$$
\mathcal{H}(Y)=Y-F^{*} Y F+F^{*} Y G\left(R+G^{*} Y G\right)^{-1} G^{*} Y F=0,
$$

where $R$ is nonsingular. Set $\Gamma=G R^{-1} G^{*}$. Then

$$
F_{Y}=F-G\left(R+G^{*} Y G\right)^{-1} G^{*} Y F=(I+\Gamma Y)^{-1} F,
$$

and we have $\mathcal{H}(Y)=Y-F^{*} Y F_{Y}=0$. We first note two results on nonsingular solutions of (4.1).

Lemma 4.1 (see [26, p. 931]). Let $Y$ be a hermitian nonsingular $n \times n$ matrix. Assume that $F$ is nonsingular. Then $Y$ is a solution of (4.1) if and only if $W=Y^{-1}$ satisfies the discrete-time Lyapunov equation

$$
W-F W F^{*}=-\Gamma .
$$

Lemma 4.2 (see [26, p. 932]). Assume that $F$ is nonsingular, $(F, G)$ is controllable, $R>0$, and $\sigma(F) \cap \sigma\left(F^{-*}\right)=\emptyset$. Then (4.1) has a unique nonsingular solution.

The subsequent result deals with eigenvalues of $F$ in $\{0\} \cup \partial \mathbb{D}$ and corresponding generalized eigenspaces.

Lemma 4.3 (see [26, p. 933]). Assume $R>0$ and $\operatorname{rank}(F-\eta I, G)=n$ for all $\eta \in \partial \mathbb{D}$. If $Y$ is a solution of (4.1), then $E_{0}(F)+E_{\partial \mathbb{D}}(F) \subseteq \operatorname{Ker} Y$.

Corollary 4.4 (see [26, pp. 923-924]). Assume $R>0$, $\operatorname{rank}[F-\lambda I, G]=n$ if $\lambda \neq 0$, and $\sigma(F) \cap \sigma(F)^{\nabla} \subseteq \partial \mathbb{D}$. Then (4.1) has a unique solution $\Delta$ with Ker $\Delta=$ $E_{0}(F)+E_{\partial \mathbb{D}}(F)$. 
It is easy to show (see also Proposition 5.2) that $(0, \Delta)$ is a pair of opposite unmixed solutions of $\mathcal{H}(Y)=0$. Therefore, the following result can already be viewed as a special case of the main theorem.

THEOREM 4.5. The assumptions on the DARE

$$
\mathcal{H}(Y)=Y-F^{*} Y F+F^{*} Y G\left(R+G^{*} Y G\right)^{-1} G^{*} Y F=0
$$

are the following: $R>0, \sigma(F) \cap \sigma(F)^{\nabla} \subseteq \partial \mathbb{D}$, and $\operatorname{rank}[F-\lambda I, G]=n$ if $\lambda \neq 0$. Define

$$
K=E_{0}(F)+E_{\partial \mathbb{D}}(F),
$$

and

$$
\mathcal{T}=\{Y \mid \mathcal{H}(Y)=0\}, \quad \mathcal{N}=\{N \in \operatorname{Inv} F \mid K \subseteq N\} .
$$

Let $\Delta$ be the solution of (4.1) with Ker $\Delta=K$. Then the following holds.

(i) If $Y$ is a solution of (4.1), then $\operatorname{Ker} Y \in \mathcal{N}$.

(ii) If $N \in \mathcal{N}$, then

$$
\mathbb{C}^{n}=(\Delta N)^{\perp} \oplus(N \cap \operatorname{Im} \Delta),
$$

and if $P_{N}$ is the projection of $\mathbb{C}^{n}$ on $(\Delta N)^{\perp}$ along $N \cap \operatorname{Im} \Delta$, then $\tilde{Y}=\Delta P_{N}$ is the unique solution of (4.1) with $\operatorname{Ker} \tilde{Y}=N$.

(iii) For $N \in \mathcal{N}$ set $\tilde{\kappa}(N)=\Delta P_{N}$. Then the map $\tilde{\kappa}: \mathcal{N} \rightarrow \mathcal{T}$ is a bijection, and for $Y \in \mathcal{T}$ we have $\tilde{\kappa}^{-1}(Y)=\operatorname{Ker} Y$.

Proof. Let us first prove the theorem under the stronger assumption that $F$ is nonsingular and $\sigma(F) \cap \sigma\left(F^{-*}\right)=\emptyset$. In that case we have $K=0$ and the solution $\Delta$ is nonsingular such that $N \cap \operatorname{Im} \Delta=N$ and $\mathcal{N}=\operatorname{Inv} F$.

(i) This is obvious since $\mathcal{H}(Y)=Y-F^{*} Y F_{Y}=0$ is equivalent to $F_{Y}^{-*} Y=Y F$.

(ii) Assume $N=\operatorname{Im}\left[\begin{array}{ll}I O\end{array}\right]^{T}$ such that

$$
F=\left[\begin{array}{cc}
F_{1} & * \\
0 & F_{2}
\end{array}\right] \text {. }
$$

It is known from Lemma 4.1 that $W=\Delta^{-1}$ satisfies

$$
W-F W F^{*}=-G R^{-1} G^{*} .
$$

Let

$$
\Delta=\left[\begin{array}{cc}
\Delta_{1} & \Delta_{21}^{*} \\
\Delta_{21} & \Delta_{2}
\end{array}\right], G=\left[\begin{array}{c}
* \\
G_{2}
\end{array}\right], \text { and } \Delta^{-1}=W=\left[\begin{array}{cc}
* & * \\
* & W_{2}
\end{array}\right]
$$

be partitioned according to (4.5). To establish a decomposition

$$
\mathbb{C}^{n}=(\Delta N)^{\perp} \oplus N,
$$

we recall Lemma 3.1 and the fact that (4.7) holds if and only if $\Delta_{1}$ is nonsingular. Thus, according to Lemma 3.2 we have to show that $W_{2}$ is nonsingular. From (4.6) we obtain

$$
W_{2}-F_{2} W_{2} F_{2}^{*}=-G_{2} R^{-1} G_{2}^{*}
$$


In the preceding Stein equation the pair $\left(F_{2}, G_{2}\right)$ is controllable, $\sigma\left(F_{2}\right) \cap \sigma\left(F_{2}^{-*}\right)=\emptyset$, and $R>0$. Therefore (see, e.g., [14, p. 453]) the matrix $W_{2}$ is nonsingular.

Now let us show that $\tilde{Y}=\Delta P_{N}$ is a solution of (4.1). Since $\Delta$ is assumed to be nonsingular we have $\operatorname{Ker} \tilde{Y}=\operatorname{Ker} P_{N}=N$. Hence it follows from Lemmas 3.1 and 3.2 that $\tilde{Y}=P_{N}^{*} \Delta P_{N}=\operatorname{diag}\left(0, \widetilde{\Delta}_{2}\right)$, and $\tilde{\Delta}_{2}=\Delta_{2}-\Delta_{21} \Delta_{1}^{-1} \Delta_{21}^{*}$ is nonsingular and $\widetilde{\Delta}_{2}^{-1}=W_{2}$. Consider the equation

$$
\mathcal{H}_{2}\left(Y_{2}\right)=Y_{2}-F_{2}^{*} Y_{2} F_{2}+F_{2}^{*} Y_{2} G_{2}\left(R+G_{2}^{*} Y_{2} G_{2}\right)^{-1} G_{2}^{*} Y_{2} F_{2}
$$

It is easy to check that $\mathcal{H}(\tilde{Y})=\operatorname{diag}\left(0, \mathcal{H}_{2}\left(\tilde{\Delta}_{2}\right)\right)$. Hence $\tilde{Y}$ is a solution of (4.1) if and only if $\mathcal{H}_{2}\left(\widetilde{\Delta}_{2}\right)=0$. To show that $\widetilde{\Delta}_{2}$ is a solution of (4.9) we use Lemma 4.1 again, which says that $\mathcal{H}_{2}\left(\widetilde{\Delta}_{2}\right)=0$ is equivalent to

$$
\widetilde{\Delta}_{2}^{-1}-F_{2} \widetilde{\Delta}_{2}^{-1} F_{2}^{*}=-G_{2} R^{-1} G_{2}^{*} .
$$

We have seen that the solution $\Delta$ gives rise to (4.8). Therefore, because $W_{2}=\widetilde{\Delta}_{2}^{-1}$, (4.10) is satisfied.

To prove uniqueness, take a solution $Y$ of (4.1) with $\operatorname{Ker} Y=N$. Then $Y=$ $\operatorname{diag}\left(0, Y_{2}\right)$, and $Y_{2}$ is nonsingular satisfying (4.9). According to Lemma 4.2 there exists a unique nonsingular solution of (4.9), namely, $\widetilde{\Delta}_{2}$. Hence $Y=\operatorname{diag}\left(0, \widetilde{\Delta}_{2}\right)=\tilde{Y}$. Part (iii) is an obvious consequence of (i) and (ii).

We now discard the assumptions about $F$ made at the beginning and consider the case $K=E_{0}(F)+E_{\partial \mathbb{D}}(F) \neq 0$. Let $V=\oplus\left\{E_{\lambda}(F) \mid \lambda \neq 0, \lambda \notin \partial \mathbb{D}\right\}$ be the $F$-invariant complement of $K$ such that $\mathbb{C}^{n}=K \oplus V$. Suppose

$$
K=\operatorname{Im}\left[\begin{array}{c}
I_{n_{a}} \\
0
\end{array}\right] \text { and } V=\operatorname{Im}\left[\begin{array}{c}
0 \\
I_{n_{c}}
\end{array}\right]
$$

such that

$$
F=\left[\begin{array}{cc}
F_{a} & 0 \\
0 & F_{c}
\end{array}\right], G=\left[\begin{array}{c}
* \\
G_{c}
\end{array}\right]
$$

Then $\sigma\left(F_{a}\right) \subseteq\{0\} \cup \partial \mathbb{D}, F_{c}$ is nonsingular, and $\sigma\left(F_{c}\right) \cap \sigma\left(F_{c}^{-*}\right)=\emptyset$. It follows from Lemma 4.3 that $Y$ is a solution of (4.1) if and only if $Y=\operatorname{diag}\left(0, Y_{c}\right)$ and $Y_{c}$ is a solution of

$$
\mathcal{H}_{c}\left(Y_{c}\right)=Y_{c}-F_{c}^{*} Y_{c} F_{c}+F_{c}^{*} Y_{c} G_{c}\left(R+G_{c}^{*} Y_{c} G_{c}\right)^{-1} G_{c}^{*} Y_{c} F_{c}=0 .
$$

Set $\mathcal{T}_{c}=\left\{Y_{c} \mid \mathcal{H}_{c}\left(Y_{c}\right)=0\right\}$ and let $\iota: \mathcal{T}_{c} \rightarrow \mathcal{T}$ be the bijection given by $\iota\left(Y_{c}\right)=$ $\operatorname{diag}\left(0, Y_{c}\right)$. Let $\Delta_{c}$ be the unique nonsingular solution of (4.12). Then $\Delta=\operatorname{diag}\left(0, \Delta_{c}\right)$ is the unique solution of (4.1) with $\operatorname{Ker} \Delta=K$.

Let $\hat{N}_{c} \in \operatorname{Inv} F_{c}$. We embed $\hat{N}_{c}$ into the space

$$
N_{c}=\left\{\left[\begin{array}{c}
0 \\
x_{c}
\end{array}\right] \in \mathbb{C}^{n}, x_{c} \in \hat{N}_{c}\right\}
$$

and define $\tau\left(\hat{N}_{c}\right)=K \oplus N_{c}$. Then $\tau: \operatorname{Inv} F_{c} \rightarrow \mathcal{N}$ is a bijection. In accordance with (4.7) we have

$$
\mathbb{C}^{n_{c}}=\left(\Delta_{c} \hat{N}_{c}\right)^{\perp} \oplus \hat{N}_{c}
$$


which is equivalent to $V=\left(\Delta_{c} N_{c}\right)^{\perp_{c}} \oplus N_{c}$. Hence, it follows from Lemma 3.3 that $N=\tau\left(\hat{N}_{c}\right) \in \mathcal{N}$ satisfies (4.4). Let $P_{\hat{N}_{c}}$ be the projection corresponding to (4.13) and set $\tilde{\kappa}_{c}\left(\hat{N}_{c}\right)=\Delta_{c} P_{\hat{N}_{c}}$. We know that $\tilde{\kappa}_{c}: \operatorname{Inv} F_{c} \rightarrow \mathcal{T}_{c}$ is a bijection. Define $\mu_{c}=\tilde{\kappa}_{c}^{-1}$, i.e., $\mu_{c}\left(Y_{c}\right)=\operatorname{Ker} Y_{c}, Y_{c} \in \mathcal{T}_{c}$. The maps $\tilde{\kappa}_{c}$ and $\mu_{c}$ can now be extended to bijections between $\mathcal{T}$ and $\mathcal{N}$. For $Y \in \mathcal{T}$ define $\mu(Y)=\operatorname{Ker} Y$. Then $\mu=\tau \mu_{c} \iota^{-1}: \mathcal{T} \rightarrow \mathcal{N}$, and $\mu$ is bijective. Let $P_{N}$ be the projection arising from (4.4). For $N \in \mathcal{N}$ define $\tilde{\kappa}(N)=\Delta P_{N}$. In the setting of (4.11) we have $P_{N}=\operatorname{diag}\left(I, P_{\hat{N}_{c}}\right)$. Therefore

$$
\tilde{\kappa}(N)=\operatorname{diag}\left(0, \Delta_{c} P_{\hat{N}_{c}}\right)=\iota\left(\Delta_{c} P_{\hat{N}_{c}}\right)=\iota \tilde{\kappa}_{c}\left(\hat{N}_{c}\right)=\iota \tilde{\kappa}_{c} \tau^{-1}(N) .
$$

Hence $\tilde{\kappa}: \mathcal{N} \rightarrow \mathcal{T}$ and $\tilde{\kappa}=\mu^{-1}$.

In order to characterize unmixed solutions of $\mathcal{D}(X)=0$, we need a discrete-time counterpart of a result of Scherer [22, p. 106]. Let $A \in \mathbb{C}^{n \times n}$ and $M \in \operatorname{Inv} A$; let $\bar{A}$ denote the endomorphism of $\mathbb{C}^{n} / M$ induced by $A$; and let $A_{\mid M}$ be the restriction of $A$ to $M$. We define $\sigma_{i}(A, M)=\sigma\left(A_{\mid M}\right)$ and $\sigma_{o}(A, M)=\sigma(\bar{A})$. Assumptions and definitions in the lemma below are those of Theorem 4.5 and its proof.

Lemma 4.6. For $N \in \mathcal{N}$ let $Y=\Delta P_{N}$ be the corresponding solution of (4.1). (i) Then $F=F_{Y}$ on $N$ and $N \in \operatorname{Inv} F_{Y}$, and we have

$$
\sigma_{i}\left(F_{Y}, N\right)=\sigma_{i}(F, N) \text { and } \sigma_{o}\left(F_{Y}, N\right)=\sigma_{o}(F, N)^{\nabla} .
$$

(ii) The solution $Y$ is unmixed if and only if $N \in \mathcal{N}$ is a spectral subspace of $F$.

Proof. Let us assume for simplicity that $F$ is nonsingular and $\sigma(F) \cap \sigma\left(F^{-*}\right)=\emptyset$.

(i) As before, suppose $N=\operatorname{Im}\left[\begin{array}{ll}I & O\end{array}\right]^{T}$ and let $F$ be given by (4.5). Then $\sigma_{i}(F, N)=\sigma\left(F_{1}\right)$ and $\sigma_{o}(F, N)=\sigma\left(F_{2}\right)$. From $Y=\Delta P_{N}=\operatorname{diag}\left(0, \widetilde{\Delta}_{2}\right)$ follows

$$
F_{Y}=(I+\Gamma Y)^{-1} F=\left[\begin{array}{cc}
F_{1} & * \\
0 & A_{2}
\end{array}\right]
$$

with $A_{2}=\left(I+\Gamma_{2} \widetilde{\Delta}_{2}\right)^{-1} F_{2}$. Thus $\sigma_{i}\left(F_{Y}, N\right)=\sigma\left(F_{1}\right)$. On the other hand, $Y-$ $F^{*} Y F_{Y}=0$ implies $A_{2}=\widetilde{\Delta}_{2}^{-1} F_{2}^{-*} \widetilde{\Delta}_{2}$, and we obtain $\sigma\left(A_{2}\right)=\sigma\left(F_{2}\right)^{\nabla}$.

(ii) $N$ is a spectral subspace of $F$ if and only if

$$
\sigma\left(F_{1}\right) \cap \sigma\left(F_{2}\right)=\emptyset .
$$

The assumption $\sigma(F) \cap \sigma\left(F^{-*}\right)=\emptyset$ together with (4.14) imply

$$
\begin{aligned}
& \sigma\left(F_{Y}\right) \cap \sigma\left(F_{Y}^{-*}\right)=\left[\sigma\left(F_{1}\right) \cup \sigma\left(F_{2}^{-*}\right)\right] \cap\left[\sigma\left(F_{1}^{-*}\right) \cup \sigma\left(F_{2}\right)\right]= \\
& {\left[\sigma\left(F_{2}^{-*}\right) \cap \sigma\left(F_{1}^{-*}\right)\right] \cup\left[\sigma\left(F_{1}\right) \cap \sigma\left(F_{2}\right)\right]=\left[\sigma\left(F_{1}\right) \cap \sigma\left(F_{2}\right)\right]^{\nabla} \cup\left[\sigma\left(F_{1}\right) \cap \sigma\left(F_{2}\right)\right] .}
\end{aligned}
$$

Hence, the property that $Y$ is unmixed, i.e., $\sigma\left(F_{Y}\right) \cap \sigma\left(F_{Y}^{-*}\right)=\emptyset$, is equivalent to (4.15).

5. The main result. The passage from the general DARE

$$
\mathcal{D}(X)=X-F^{*} X F+\left(G^{*} X F+S\right)^{*}\left(R+G^{*} X G\right)^{-1}\left(G^{*} X F+S\right)-Q=0
$$

to an equation of the form $\mathcal{H}(Y)=0$ in (4.1) is a crucial step in the derivation of our main theorem. It is based on the following lemma for which we refer to [19] and [8, Lemma 5.2] or [1, Lemma 6.8.9]. 
Lemma 5.1. (i) Let $X_{2}$ be a solution of $\mathcal{D}(X)=0$. Then $X$ is a solution of $\mathcal{D}(X)=0$ if and only if $Y=X-X_{2}$ is a solution of

$$
\mathcal{H}_{2}(Y)=Y-F_{X_{2}}^{*} Y F_{X_{2}}+F_{X_{2}}^{*} Y G\left[\left(R+G^{*} X_{2} G\right)+G^{*} Y G\right]^{-1} G^{*} Y F_{X_{2}}=0 .
$$

(ii) Let $X_{2}$ and $X$ be solutions of $\mathcal{D}(X)=0$. Set $Y=X-X_{2}$. Then

$$
F_{X}=F_{X_{2}}-G\left(R+G^{*} X G\right)^{-1} G^{*} Y F_{X_{2}} .
$$

Thus, when a solution $X_{2}$ of (5.1) is at our disposal we can pass from (5.1) to (5.2) and apply the results of section 4 .

Proposition 5.2. Assume $(\Psi)$ and

$$
\operatorname{rank}[F-\lambda I, G]=n \text { if } \lambda \neq 0 .
$$

Let $X_{2}$ be an unmixed solution of $\mathcal{D}(X)=0$. Set $K=E_{0}\left(F_{X_{2}}\right)+E_{\partial \mathbb{D}}\left(F_{X_{2}}\right)$. Then there exists a unique solution $\Delta$ of $(5.2)$ with $\operatorname{Ker} \Delta=K$. Moreover $\left(X, X_{2}\right)$ is a pair of opposite unmixed solutions of $\mathcal{D}(X)=0$ if and only if $X=X_{2}+\Delta$.

Proof. The assumption ( $\Psi$ ) implies $R+G^{*} X_{2} G>0$ in (5.2). Clearly (5.4) implies the corresponding condition for $F=F_{X_{2}}$. The assumption that $X_{2}$ should be an unmixed solution of (5.1) is equivalent to

$$
\sigma\left(F_{X_{2}}\right) \cap \sigma\left(F_{X_{2}}\right)^{\nabla} \subseteq \partial \mathbb{D} .
$$

Hence it follows from Corollary 4.4 that (5.2) has a unique solution $\Delta$ with $\operatorname{Ker} \Delta=$ $K$. Again it is no loss of generality to assume

$$
K=\operatorname{Im}\left[\begin{array}{c}
I_{n-n_{c}} \\
0
\end{array}\right]
$$

and $F_{X_{2}}=\operatorname{diag}\left(F_{a}, F_{c}\right)$. Then $\Delta=\operatorname{diag}\left(0, \Delta_{c}\right)$, det $\Delta_{c} \neq 0$. Obviously (5.5) implies that $F_{c}$ is nonsingular and

$$
\sigma\left(F_{c}\right) \cap \sigma\left(F_{c}^{-*}\right)=\emptyset .
$$

Let $X$ be a solution of (5.1). Set $Y=X-X_{2}$. Then $\mathcal{H}_{2}(Y)=0$, or equivalently

$$
Y-F_{X_{2}}^{*} Y F_{X}=0 .
$$

Because of Lemma 4.3 we have $K \subseteq \operatorname{Ker} Y$. Hence $Y=\operatorname{diag}\left(0, Y_{c}\right)$. The identity (5.3) yields

$$
F_{X}=\left[\begin{array}{cc}
F_{a} & * \\
0 & B
\end{array}\right]
$$

From (5.7) we obtain

$$
Y_{c}-F_{c}^{*} Y_{c} B=0 .
$$

Let us consider the solution $X=X_{2}+\Delta$ and let $F_{X}$ be as in (5.8). In this case we have $Y=\Delta=\operatorname{diag}\left(0, \Delta_{c}\right)$. Thus (5.9) becomes $\Delta_{c}-F_{c}^{*} \Delta_{c} B=0$, where $F_{c}$ and $\Delta_{c}$ are nonsingular. Hence $B=\Delta_{c}^{-1} F_{c}^{-*} \Delta_{c}, \sigma(B)=\sigma\left(F_{c}^{-*}\right)$, and

$$
\sigma\left(F_{X}\right)=\sigma\left(F_{a}\right) \cup \sigma(B)=\sigma\left(F_{a}\right) \cup \sigma\left(F_{c}^{-*}\right) .
$$


Thus (5.6) implies $\sigma\left(F_{X}\right) \cap \sigma\left(F_{X_{2}}\right)=\sigma\left(F_{a}\right) \subseteq\{0\} \cup \partial \mathbb{D}$, and therefore $\left(X, X_{2}\right)$ is a pair of opposite unmixed solutions.

Now consider a solution $X$ such that $Y=X-X_{2} \neq \Delta$. Then $Y=\operatorname{diag}\left(0, Y_{c}\right)$ and $Y_{c}$ is singular. Take $w \in \operatorname{Ker} Y_{c}, w \neq 0$. Recall that $F_{c}$ in (5.9) is nonsingular. Hence $F_{c} B w=0$, and $\operatorname{Ker} Y_{c}$ is invariant under $B$. Therefore we can assume that $w$ is an eigenvector of $B$, say $B w=\lambda w$. Interchanging the role of $X$ and $X_{2}$ in (5.3), we obtain

$$
F_{X_{2}}=\operatorname{diag}\left(F_{a}, F_{c}\right)=F_{X}-G\left(R+G^{*} X_{2} G\right)^{-1} G^{*}(-Y) F_{X} .
$$

Hence $F_{c}=B-W Y_{c} B$ with some $n_{c} \times n_{c}$ matrix $W$. Thus $F_{c} w=B w$. Therefore $\lambda \in \sigma\left(F_{c}\right)$, and $\lambda \notin(\{0\} \cup \partial \mathbb{D})$. On the other hand $\lambda \in \sigma\left(F_{X}\right) \cap \sigma\left(F_{X_{2}}\right)$. Hence $\left(X, X_{2}\right)$ is not a pair of opposite unmixed solutions.

At this point the pieces can be put together.

THEOREM 5.3. Let $\left(X_{1}, X_{2}\right)$ be a pair of opposite unmixed solutions of the DARE $\mathcal{D}(X)=0$ in (5.1). Set $\Delta=X_{1}-X_{2}$ and

$$
F_{X_{2}}=F-G\left(R+G^{*} X_{2} G\right)^{-1}\left(G^{*} X_{2} F+S\right) .
$$

Let $\mathcal{S}$ be the set of hermitian solutions of $\mathcal{D}(X)=0$, and define

$$
\mathcal{N}=\left\{N \in \operatorname{Inv} F_{X_{2}} \mid E_{0}\left(F_{X_{2}}\right)+E_{\partial \mathbb{D}}\left(F_{X_{2}}\right) \subseteq N\right\} .
$$

Assume $(\Psi)$ and $\operatorname{rank}[F-\lambda I, G]=n$ if $\lambda \neq 0$. If $N \in \mathcal{N}$, then

$$
(\Delta N)^{\perp} \oplus(N \cap \operatorname{Im} \Delta)=\mathbb{C}^{n} .
$$

Let $P_{N}$ be the projection on $(\Delta N)^{\perp}$ along $N \cap \operatorname{Im} \Delta$. Define

$$
\kappa(N)=X_{1} P_{N}+X_{2}\left(I-P_{N}\right) .
$$

Then $\kappa: \mathcal{N} \rightarrow \mathcal{S}$ is a bijection. If $X \in \mathcal{S}$, then

$$
\kappa^{-1}(X)=\operatorname{Ker}\left(X-X_{2}\right) .
$$

Proof. Let $\mathcal{T}_{2}$ denote the set of hermitian solutions of $\mathcal{H}_{2}(Y)=0$ in (5.2). By Lemma 5.1 we have $X \in \mathcal{S}$, if and only if $Y=X-X_{2} \in \mathcal{T}_{2}$. Hence $\mathcal{S}=X_{2}+\mathcal{T}_{2}$. If $\left(X_{1}, X_{2}\right)$ is a pair of opposite unmixed solutions, then it follows from Proposition 5.2 that $\Delta=X_{1}-X_{2}$ is the solution of (5.2) with Ker $\Delta=E_{0}\left(F_{X_{2}}\right)+E_{\partial \mathbb{D}}\left(F_{X_{2}}\right)$. Hence we can parametrize the set $\mathcal{T}_{2}$ according to Theorem 4.5. If we set $\tilde{\kappa}(N)=\Delta P_{N}$, then $\tilde{\kappa}: \mathcal{N} \rightarrow \mathcal{T}_{2}$ is a bijection with $\tilde{\kappa}^{-1}(Y)=\operatorname{Ker} Y$. Thus $\mathcal{S}=X_{2}+\mathcal{T}_{2}$ gives rise to a bijection $\kappa: \mathcal{N} \rightarrow \mathcal{S}$ defined by $\kappa(N)=X_{2}+\tilde{\kappa}(N)$. Then $\kappa(N)=X_{2}+\left(X_{1}-X_{2}\right) P_{N}$ yields (5.12). Finally, for $X \in \mathcal{S}$ we have $\kappa^{-1}(X)=\tilde{\kappa}^{-1}\left(X-X_{2}\right)=\operatorname{Ker}(X-$ $\left.X_{2}\right)$.

Corollary 5.4. A solution $X$ of (5.1) is unmixed if and only if $\operatorname{Ker}\left(X-X_{2}\right)$ is a spectral subspace of $F_{X_{2}}$.

Proof. Let $X$ be a solution of (5.1) and set $Y=X-X_{2}$ such that $Y$ is a solution of $\mathcal{H}_{2}(Y)=0$ in (5.2). We claim that $X$ is an unmixed solution of (5.1) if and only if the corresponding matrix $Y$ is an unmixed solution of (5.2). Let $A$ be the closed loop matrix associated with $Y$ (with respect to $\mathcal{H}_{2}=0$ ), i.e.,

$$
A=F_{X_{2}}-G\left[\left(R+G^{*} X_{2} G\right)+G^{*} Y G\right]^{-1} G^{*} Y F_{X_{2}} .
$$


Now the identity (5.3) shows that $A=F_{X}$. We can apply Lemma 4.6, which tells us that $Y$ is an unmixed solution of (5.2) if and only if $N=\tilde{\kappa}^{-1}(Y)=\operatorname{Ker} Y$ is a spectral subspace of $F_{X_{2}}$.

The pair of opposite solutions $\left(X_{-}, X_{+}\right)$satisfying (2.11) consists of the smallest and the greatest solution of (5.1), i.e., for each $X \in \mathcal{S}$ we have $X_{-} \leq X \leq X_{+}$. In that particular case, Theorem 5.3 can be obtained from a result on intervals of solutions in [27].

\section{REFERENCES}

[1] H. Abou-Kandil, G. Freiling, V. Ionescu, and G. Jank, Matrix Riccati Equations in Control and Systems Theory, Birkhäuser, Basel, 2003.

[2] W. N. Anderson, JR., Shorted operators, SIAM J. Appl. Math., 20 (1971), pp. 520-525.

[3] D. J. Clements And H. K. Wimmer, Existence and uniqueness of unmixed solutions of the discrete-time algebraic Riccati equation, Systems Control Lett., 50 (2003), pp. 343-346.

[4] A. N. Churilov, On solutions of a quadratic matrix equation encountered in the investigation of discrete control systems, Sov. Math. (Iz. VUZ), 30 (1986), pp. 81-89.

[5] W. A. Coppel, Matrix quadratic equations, Bull. Austral. Math. Soc., 10 (1974), pp. 377-401.

[6] G. Corach, A. Maestripieri, and D. Stojanoff, Oblique projections and Schur complements, Acta Sci. Math. (Szeged), 67 (2001), pp. 337-356.

[7] A. Ferrante and H. K. Wimmer, Order reduction of discrete-time algebraic Riccati equations with singular closed loop matrix, Eur. J. Control, submitted.

[8] G. Freiling and A. Hochhaus, Properties of the solutions of rational matrix difference equations, Comput. Math. Appl., 45 (2003), pp. 1137-1154.

[9] A. H. W. GEeRTs, The algebraic Riccati equation and singular optimal control: The discretetime case, in Systems and Networks: Mathematical Theory and Applications, Proceedings of the International Symposium MTNS 93, Regensburg, 1993, Vol. II, U. Helmke et al., eds., Akademie Verlag, Berlin, 1994, pp. 129-134.

[10] R. A. Horn and Ch. R. Johnson, Matrix Analysis, Cambridge University Press, New York, 1985.

[11] V. IOnEscu And C. OARĂ, Generalized discrete-time Riccati theory, SIAM J. Control Optim., 34 (1996), pp. 601-619.

[12] V. KuČERA, Algebraic Riccati equations: Hermitian and definite solutions, in The Riccati Equation, Commun. Control Engrg., S. Bittanti et al., eds., Springer-Verlag, Berlin, 1991, pp. 53-88.

[13] P. Lancaster and L. Rodman, Algebraic Riccati Equations, Clarendon Press, Oxford, UK, 1995.

[14] P. Lancaster and M. Tismenetsky, The Theory of Matrices, Academic Press, San Diego, 1985.

[15] V. Mehrmann, A step towards a unified treatment of continuous and discrete time control problems, Linear Algebra Appl., 241-243 (1996), pp.749-779.

[16] S. K. Mitra And M. L. Puri, Shorted matrices - an extended concept and some applications, Linear Algebra Appl., 42 (1982), pp. 57-79.

[17] B. P. MOLINARI, The stabilizing solution of the discrete algebraic Riccati equation, IEEE Trans. Automat. Control, 20 (1975), pp. 396-399.

[18] M. Pavon And H. K. Wimmer, Suboptimal Markovian smoothing estimates based on continuous curves of solutions of the algebraic Riccati inequality, Automatica J. IFAC, 38 (2002), pp. 1017-1025.

[19] A. C. M. Ran And H. L. Trentelman, Linear quadratic problems with indefinite cost for discrete time systems, SIAM J. Matrix Anal. Appl., 14 (1993), pp. 776-797.

[20] G. Ruckebusch, Représentations Markoviennes de processus Gaussiens stationaires, C.R. Acad. Sci. Paris Ser. A, 282 (1976), pp. 649-651.

[21] G. Ruckebusch, Représentations Markoviennes de processus Gaussiens stationaires et applications statistiques, in Journées de Statistique des Processus Stochastiques, Proceedings, Grenoble 1977, Lecture Notes in Math. 636, D. Dacunha-Castelle and B. Van Cutsem, eds., Springer, Berlin, 1978, pp. 115-139.

[22] C. SCHERER, The solution set of the algebraic Riccati equation and the algebraic Riccati inequality, Linear Algebra Appl., 153 (1991), pp. 99-122.

[23] M. A. Shayman, Geometry of the algebraic Riccati equation, Part I, SIAM J. Control Optim., 21 (1983), pp. 375-394. 
[24] J. C. Willems, Least-squares stationary optimal control and the algebraic Riccati equation, IEEE Trans. Automat. Control, 16 (1971), pp. 621-634.

[25] H. K. Wimmer, Unmixed solutions of the discrete-time algebraic Riccati equation, SIAM J. Control Optim., 30 (1992), pp. 867-878.

[26] H. K. Wimmer, Hermitian solutions of the discrete-time algebraic Riccati equation, Internat. J. Control, 63 (1996), pp. 921-936.

[27] H. K. Wimmer, Intervals of solutions of the discrete-time algebraic Riccati equation, Systems Control Lett., 36 (1999), pp. 207-212. 